\title{
In-situ Electron Microscopy of Dynamic Interfaces in Heterogeneous Catalysis
}

\section{$\underline{\text { Marc Willinger }}$}

Scientific Center of Optical and Electron Microscopy, ETH Zürich, 8093 Zürich, Switzerland.

Heterogeneous catalysis is concerned with the study of reactions and dynamics at interfaces. The active catalyst is involved in bond breaking and bond making and thus, experiences a constant change in surface coverage due to adsorption, reaction, and desorption of species. The transfer of charge, energy and chemical species at the surface of the catalyst induces a response that eventually feeds back into the reaction. As a consequence, concerted dynamics emerge in the interaction between reactive gas-phase and active catalyst. They give rise to complex spatio-temporal dynamics, oscillatory behavior, and ideally, sustained catalytic activity.

Our aim is to understand processes that lead to the emergence of catalytic function though direct observation using a combination of operando scanning and transmission electron microscopy.

Starting with simple model catalysts, such as polycrystalline metal foils, we observe reactions at low pressure and increase the complexity step-wise. Following this approach, we were recently able to visualize the propagation of chemical waves on the surface of polycrystalline platinum foils during $\mathrm{NO}_{2}$ hydrogenation. These experiments, performed inside the chamber of an environmental SEM, show how catalytic activity depends on grain orientation and reaction conditions, the action of "chemical clocks" and the overall behavior of an excitable system [1].

Moving from platinum to less-noble metals, we observed several redox-reactions in which the active catalyst is operating near a phase-boundary in which metallic and oxidized phase co-exist [2]. Here, we observe fascinating dynamics that increase in complexity with increasing chemical potential of the gasphase. Finally, we move up from simple model catalysts to industrially relevant metal nanoparticles that are supported on a reducible oxide carrier. Here again, we observe fascination dynamics at interfaces that can only be observed when the catalyst is in a working state. Overall, our work highlights that active catalysts are dynamically adapting to the reaction environment and that catalytic function is related to the catalysts ability to participate in the reaction through reversible changes in its structure or (local) composition. Transient high-energy sites, phase-cooperation and frustrated phase-boundaries are playing important roles in the dance of the chemical species. Which of them are relevant for the desired selective pathway and which are required for catalytic function, but eventually undesired, needs to be further elucidated.

\section{References:}

[1] C Barroo et al, Nat Catal 3 (2020), p. 30. DOI: 10.1038/s41929-019-0395-3

[2] X Huang et al, Adv Mater (2021), 2101772. DOI: 10.1002/adma.202101772 


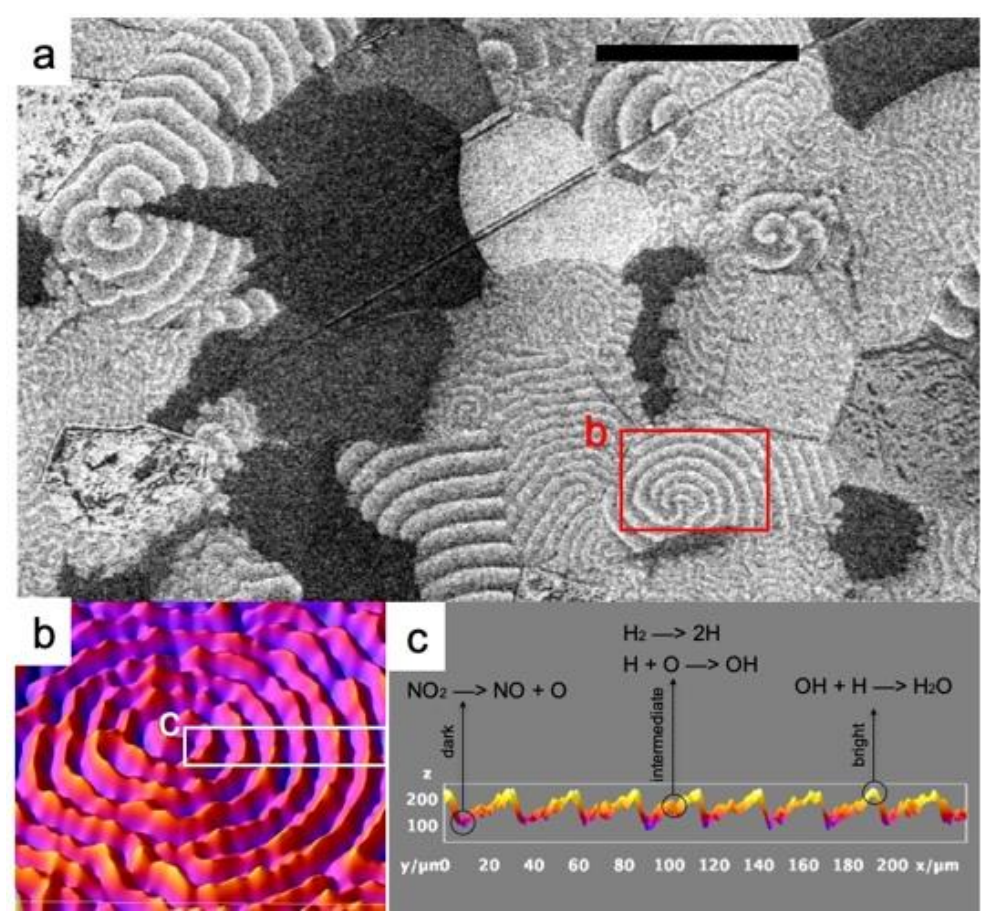

Figure 1. $\mathrm{NO}_{2}$ hydrogenation on polycrystalline Pt foils. (a) shows contrast due to propagating chemical waves and grain orientation dependent behavior. (b) Spiral wave with secondary electron signal plotted in 3D. (c) Coverage dependent contrast modulation shows threshold behavior of an excitable system.
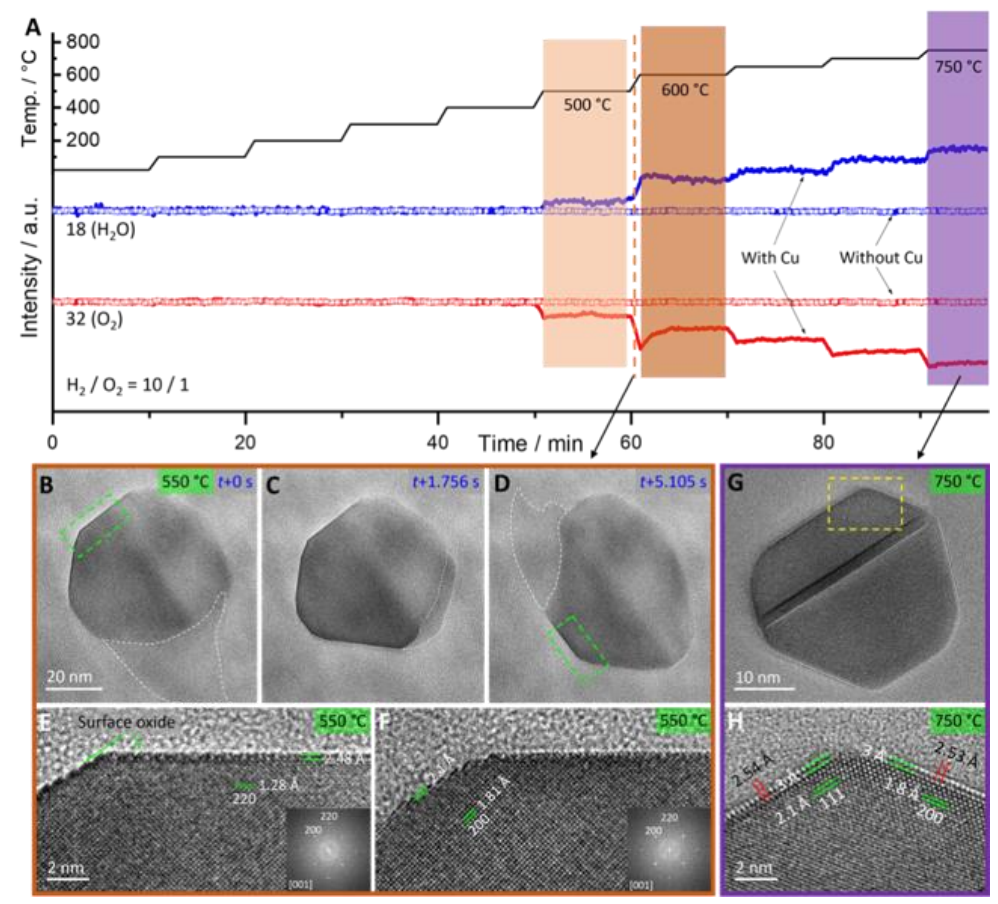

Figure 2. Copper in a $\mathrm{H}_{2} / \mathrm{O}_{2}$ redox reaction. (A) mass spectrum recorded during operando TEM observation showing the consumption of oxygen and water formation that coincides with the onset of redox dynamics of copper particles shown in (B-H). Figure reproduced with permission from Wiley. 